\title{
Controlling the emission profile of an $\mathrm{H}_{2}$ discharge lamp to simulate interstellar radiation fields
}

\author{
N. F. W. Ligterink ${ }^{\star}, 1,2$, D. M. Paardekooper ${ }^{\star}, 1$, K. -J. Chuang ${ }^{1,2}$, M. L. Both ${ }^{1}$, G. A. Cruz-Diaz ${ }^{1}$, \\ J. H. van Helden ${ }^{3}$, and H. Linnartz ${ }^{1}$
}

\author{
${ }^{1}$ Raymond and Beverly Sackler Laboratory for Astrophysics, Leiden Observatory, Leiden University, PO Box 9513, 2300 RA Leiden, \\ The Netherlands \\ e-mail: [linnartz;ligterink; dmpaardekooper]@strw.leidenuniv.nl \\ 2 Leiden Observatory, Leiden University, PO Box 9513, 2300 RA Leiden, The Netherlands \\ 3 Leibniz Institute for Plasma Science and Technology (INP Greifswald), Felix-Hausdorff-Str. 2, 17489 Greifswald, Germany
}

Received 10 July 2015 / Accepted 3 September 2015

\section{ABSTRACT}

\begin{abstract}
Context. Microwave discharge hydrogen-flow lamps have been used for more than half a century to simulate interstellar ultraviolet radiation fields in the laboratory. Recent discrepancies between identical measurements in different laboratories, as well as clear wavelength dependent results obtained in monochromatic (synchrotron) experiments, hint at a more elaborate dependence on the exact discharge settings than assumed so far.

Aims. We have investigated systematically two lamp geometries in full dependence of a large number of different running conditions and the spectral emission patterns are characterized for the first time with fully calibrated absolute flux numbers.

Methods. A sophisticated plasma lamp calibration set-up has been used to record the vacuum-ultraviolet emission spectra with a spectral resolution of $0.5 \mathrm{~nm}$ and bandwidth of $1.6 \mathrm{~nm}$ in the $116-220 \mathrm{~nm}$ region. Spectra are compared with the output of a calibrated $\mathrm{D}_{2}$-lamp which allows a derivation of absolute radiance values.

Results. The general findings of over 200 individual measurements are presented, illustrating how the lamp emission pattern depends on i) microwave power; ii) gas and gas mixing ratios; iii) discharge lamp geometry; iv) cavity positioning; and v) gas pressure.
\end{abstract}

Key words. astrochemistry - methods: laboratory: molecular - techniques: spectroscopic - molecular processes - ultraviolet: ISM

\section{Introduction}

For the last two decades, microwave discharge hydrogen-flow lamps (MDHLs; Warneck 1962; Davis \& Braun 1968) have been widely used as vacuum-ultraviolet (VUV) light sources in laboratory experiments to simulate interstellar radiation fields in astrochemical studies (Westley et al. 1995; Gerakines et al. 1996; Baratta et al. 2002; Muñoz Caro et al. 2002, 2010; Cottin et al. 2003; Leto \& Baratta 2003; Loeffler et al. 2005; Watanabe et al. 2007; Öberg et al. 2007; Islam et al. 2014; Cook et al. 2014; Henderson \& Gudipati 2015). These experiments aim to simulate and ultimately unravel the complex photoprocesses (photodesorption, photodissociation, and photochemistry) taking place in interstellar ice analogues. The spectral distribution of the interStellar radiation field (ISRF) is strongly dependent on the interstellar environment. As ice processing is particularly interesting in dark interstellar clouds which are largely shielded from external light sources, laboratory studies have been focusing on simulating the VUV photons emitted by $\mathrm{H}$ and $\mathrm{H}_{2}$ after excitation by cosmic rays that do penetrate the cloud. In the laboratory, these photons are produced using a MDHL. Typically, it has been assumed that the lamp spectrum is dominated by Lyman- $\alpha$ photons around $121 \mathrm{~nm}$, with a broad and weaker emission band situated around $160 \mathrm{~nm}$. The estimated flux of the MDHL at sample position in a number of papers is based on previously reported values in the literature, which roughly span between $10^{13}-10^{15}$ photons $\mathrm{s}^{-1} \mathrm{~cm}^{-2}$ and generally lack

\footnotetext{
* Both authors contributed equally to this work.
}

information on the spectral energy distribution (SED). However, exact running conditions of the lamp, i.e. the dependence on the experimental settings, influence both the total photon flux and the SED of the impacting radiation at the ice sample. In other situations the flux of the lamp is either directly measured using a National Institute of Standards and Technology (NIST) calibrated photodiode or indirectly by actinometry using for example the conversion of $\mathrm{O}_{2}$ to $\mathrm{O}_{3}$ (Gerakines et al. 1996; Cottin et al. 2003; Islam et al. 2014; Fulvio et al. 2014). When using a NIST calibrated photodiode for photon flux measurements, the quantum yield is known at different wavelengths. Since the photodiode is also sensitive to visible light, the contribution of visible light emitted by the MDHL has to be taken explicitly into account as well. In the actinometry case, the $\mathrm{O}_{2}$ to $\mathrm{O}_{3}$ conversion efficiency typically used is taken from gas phase experiments performed by Groth (1937). Recent experiments indicate that the quantum efficiency in the solid state is significantly lower (Cottin et al. 2003; Fulvio et al. 2014) and hint toward a wavelength dependency, although detailed wavelength dependent studies are still lacking in the literature. It is clear that both calibration methods require knowledge of the emission spectrum of the lamp to accurately determine the flux at different photon energies, which strongly depends on the experimental settings.

The importance of controlling the emission spectrum of the MDHLs has been pointed out by various recent studies (Chen et al. 2014; Es-sebbar et al. 2015). Photodesorption rates for several molecules using synchrotron radiation (Fayolle et al. 2011, 2013) as well as direct cross section measurements of the main 
constituents of interstellar ice show strong wavelength dependences in the VUV domain. (Cruz-Diaz et al. 2014a,b) Recently, Chen et al. (2014) showed that the differences in CO photodesorption rates reported by various groups over the past decade may be partly explained by differences in MDHL emission spectra. Carbon monoxide is an excellent example, since it strongly absorbs $160 \mathrm{~nm}$ photons while Lyman- $\alpha$ photons are clearly off resonance, as has been shown in independent experiments (Fayolle et al. 2011; Cruz-Diaz et al. 2014a).

The main purpose of the present study is to systematically identify the importance of various parameters for operating the MDHL: pressure, gas compositions $\left(\mathrm{H}_{2}, \mathrm{D}_{2}\right.$, addition of $\left.\mathrm{He}\right)$, microwave power, lamp geometry, pumping efficiency, and cavity positioning. The systematic approach presented here allows the optimization of lamp settings for specific experiments, using the MDHL in a more wavelength selective way. The study complements the work of Chen et al. (2014) and more recently Es-sebbar et al. (2015). The main difference between the present work and these two recent studies is that the spectral irradiance of the MDHLs is characterized absolutely, using a fully calibrated measurement procedure. This allows us to obtain the relative photon flux in full wavelength dependence.

We also list a number of very practical issues, such as the degradation of $\mathrm{MgF}_{2}$ windows that are typically used and potentially affect the overall outcome of studies in which accurate flux numbers are needed. It should be noted though, that the paper is not intended as a reference to skip calibration procedures. In the best case, the right order of magnitude can be derived from the information given here. The dependences that are described below will make it clear that flux measurements at the sample distance are necessary to guarantee that numbers depending on absolute photon fluxes are correct.

\section{Experimental}

Section 2.1 describes the microwave discharge hydrogen-flow lamp, while in Sect. 2.2 the UV/VUV spectrometer used for characterization is described. To convert the different radiation units, from irradiance to photon flux at the sample distance, an additional calibration set-up had to be constructed, which is described in Sect. 2.3. To test the implications that result from this work, in Sect. 2.4 the main characteristics of one of our UHV ice set-ups are described. The astronomical relevance of the MDHL findings is illustrated here with VUV ice irradiation experiments for different lamp settings.

\subsection{Microwave discharge hydrogen-flow lamp}

The MDHL consists of a pyrex lamp-tube and an air-cooled Sairem Evenson microwave cavity which is powered by a Sairem GMP $03 \mathrm{~K} / \mathrm{SM}$ microwave generator. The plasma is ignited using a BD-10A high frequency generator. The geometry of the lamp-tube can be either F- or T-type, as depicted in Fig. 1. This lamp-tube is connected to a set-up using an $\mathrm{UHV} \mathrm{MgF}_{2}$ window (Torr Scientific Ltd) and a custom-made connection piece. A rubber ring seals off the connection at the lamp side, the other two available ports are used for the inlet and outlet of the gas flowing through the tube. The port near the $\mathrm{MgF}_{2}$ window is typically the inlet of the gas. The system is evacuated by either a TS300 dry scroll pump or a DS-302 rotary vane pump, both manufactured by Agilent. The typical base pressure of the system obtained with the scroll pump and oil pump are $\sim 10^{-2}$ mbar and $\sim 5-10 \times 10^{-3}$ mbar, respectively. Pressure monitoring is performed at various positions. Two gauges (Agilent

\section{F-type MDHL (Side)}
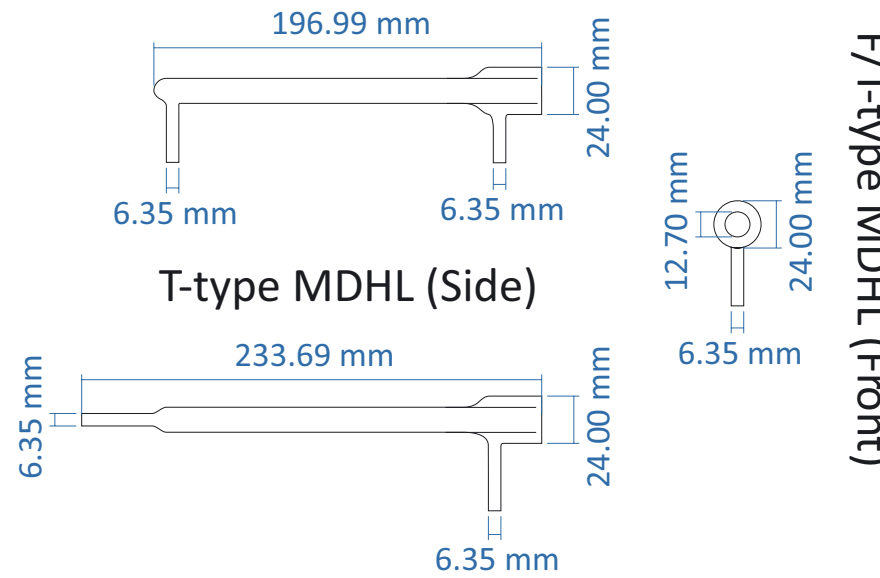

Fig. 1. Schematic of the F- and T-type MDHL used in this study. Both lamps are based on commercially available Opthos Instruments MDHLs.

PCG-750 and Granville-Phillips Convectron gauge) are situated at the inlet of the gas flow and an additional Agilent PCG750 is attached near the outlet. These gauges have been calibrated for $\mathrm{H}_{2}$ with reference to both $0.00-10.00$ mbar and 0-1000 mbar Edwards Barocell manometers with a $0.15 \%$ accuracy of reading. All the pressure values mentioned and presented are absolute values. The gas inlet of the lamp-tube can be directly connected to a needle valve, reducer, and gas bottle or to a flow controller gas mixing system. The latter can be used for pure gasses and binary gas mixtures. Flow rates in this system are given in standard cubic centimetres per minute (sccm). The gas outlet of the lamp tube is connected to a pump without further flow restrictions. Gasses used in our experiments are hydrogen (99.999\% pure, Air Liquide/Alphagaz), helium (99.999\% pure, Air Liquide/Alphagaz), and deuterium (99.8\% pure, Air Liquide/Alphagaz).

\subsection{Greifswald UVIVUV spectrometer}

All spectra presented here were measured in November 2014 during a one-week measurement campaign at the UV/VUV spectroscopy set-up located at the Leibniz Institute for Plasma Science and Technology (INP Greifswald). This setup, schematically depicted in Fig. 2, has been routinely used for the last two decades as a diagnostic tool for characterizing the UV/VUV emission of (atmospheric) plasma (Foest et al. 2007; Lange et al. 2009). The system consists of two independent optical beam lines which can be coupled into the VUV-monochromator by a shared rotatable parabolic mirror. The entire set-up is evacuated to high vacuum $\left(\sim 2 \times 10^{-6} \mathrm{mbar}\right)$ conditions by turbo molecular pumps. The MDHL is mounted onto the inlet of the first beam line, while the rest of the system is kept at high vacuum conditions by a shutter valve. The light from the lamp passes through a $\mathrm{MgF}_{2}$ window (Torr Scientific Ltd, $115 \mathrm{~nm}$ cut off) and a pinhole (0.6 mm diameter), and is directed towards the parabolic mirror. The second beam line houses a deuterium lamp (Cathodeon V03) of which the spectral radiance $\left(E_{\lambda}: \mu \mathrm{W} \mathrm{sr} \mathrm{mm}^{-1} \mathrm{~nm}^{-1}\right)$ in the $116-220 \mathrm{~nm}$ range is known; this lamp was calibrated by the Physikalisch Technische Bundesanstalt (PTB). The orientation of the rotatable parabolic mirror (focus length $1.5 \mathrm{~m}$ ) determines which of the two beam lines is optically connected to the spectrometer. The entrance slit 
N. F. W. Ligterink et al.: Controlling the emission profile of an $\mathrm{H}_{2}$ discharge lamp to simulate interstellar radiation fields

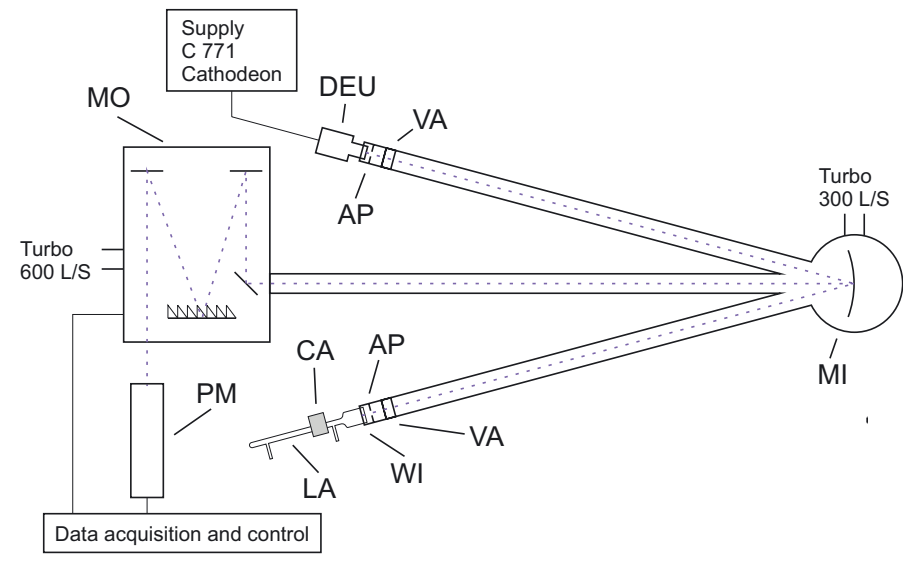

Fig. 2. Experimental set-up at the INP Greifswald for VUV measurements, (DEU): Physikalisch Technische Bundesanstalt (PTB) calibrated $\mathrm{D}_{2}$ lamp, (AP): Aperture, $0.6 \mathrm{~mm}$ (diameter), (VA): Valve, (MI): Mirror, $f=1.5 \mathrm{~m},(\mathrm{WI}): \mathrm{MgF}_{2}$ window, $(\mathrm{PM})$ : Photomultiplier, (MO): VUV-Monochromator (VM-505 Acton Research); $0.5 \mathrm{~m}, 1200 \mathrm{~g} / \mathrm{mm}$, (LA): Microwave Discharge Hydrogen-flow Lamp, (CA): Microwave Evenson cavity.

of the spectrometer is set to $1.0 \mathrm{~mm}$, giving a spectral bandwidth of $1.6 \mathrm{~nm}$. In this way the absolute response of the complete system is thus determined for a spectral bandwidth of $1.6 \mathrm{~nm}$.

The $0.5 \mathrm{~m}$ path-length spectrometer (VM-505 Acton Research) has a $1200 \mathrm{~g} / \mathrm{mm}$ grating. Light exits through a $1 \mathrm{~mm}$ slit and is detected by a VUV-photon multiplier tube. The output of the calibrated spectra is given in absolute spectral radiance as $\mu \mathrm{W} \mathrm{sr}-1 \mathrm{~mm}^{-2} \mathrm{~nm}^{-1}$.

\subsection{Photon flux measurements}

The conversion of spectral radiance $\left(\mu \mathrm{W} \mathrm{Sr}{ }^{-1} \mathrm{~mm}^{-2} \mathrm{~nm}^{-1}\right)$ to photon flux at the sample location (photon $\mathrm{cm}^{-2} \mathrm{~s}^{-1}$ ) is far from trivial. This is true even when all the dimensions of the set-up are known. In the Greifswald set-up only a small fraction of the plasma contributes to the light passing through the pinhole (diameter $0.6 \mathrm{~mm}$ ); extrapolating to the full plasma introduces a large error and assumes that the plasma is homogeneous. Therefore, a set of complimentary VUV photon flux measurements of the MDHLs at various settings have been performed on a small set-up specifically constructed for this purpose in Leiden. It consists of a calibrated photodiode, shutter valve, and $\mathrm{MgF}_{2}$ window with connection piece for the MDHL. The photodiode is a $1 \mathrm{~cm}^{2}$ NIST calibrated AXUV-100, of which the photocurrent is measured using a Keithley 485 autorange picoammeter. The distance between the $\mathrm{MgF}_{2}$ window and photodiode is $31 \mathrm{~cm}$. The entire set-up is evacuated to a pressure of $10^{-2}$ mbar. The photodiode is used to measure the total flux emitted by the MDHL. This type of photodiode has a low band gap of $1.12 \mathrm{eV}$ and is therefore also sensitive to visible light. The contribution of visible light to the photocurrent has to be subtracted. Flux measurements are thus performed with and without a $\mathrm{KBr}$ window in the optical path. This window blocks any radiation below $240 \mathrm{~nm}$ and has $90 \%$ transmission above this wavelength, up to $1000 \mathrm{~nm}$. By subtracting the visible response from the total photocurrent of the diode, corrected for the absorption of $\mathrm{KBr}$, the photocurrent in the range between 115 to $240 \mathrm{~nm}$ can be determined. This yields the total energy in this spectral range, and combined with the spectrum obtained at the calibrated set-up in Greifswald, the photocurrent per wavelength can be determined. It must be noted that the energy recorded in the range $115-240 \mathrm{~nm}$ is assumed to be equal to the energy in the range $116-220 \mathrm{~nm}$, since no hydrogen emission is present from 220 to $240 \mathrm{~nm}$. The transmission of the $\mathrm{MgF}_{2}$ window between 115 and 116 is small. The calibrated spectrum is then converted to units of photons $\mathrm{sr}^{-1} \mathrm{~mm}^{-2} \mathrm{~nm}^{-1}$ and normalized to an integrated value of one. Next, the measured total VUV photocurrent is assigned proportionally to each wavelength by the normalized spectrum. This results in a wavelength dependent photocurrent that can be converted to a wavelength dependent photon flux according to Eq. (1),

$I(\lambda)=\frac{i(\lambda)}{e \cdot \eta(\lambda)}$,

where $I(\lambda)$ is the wavelength dependent photon flux in photon $\mathrm{cm}^{-2} \mathrm{~s}^{-1}, \mathrm{i}(\lambda)$ the photocurrent, $e$ the electron charge, and $\eta(\lambda)$ the detection efficiency. The resulting wavelength dependent photon flux can be integrated to obtain the total photon flux of the lamp. Absolute Lyman- $\alpha$ and molecular emission photon fluxes are determined by integrating the 120-124 and $155-165 \mathrm{~nm}$ regions, respectively. In our spectra more emission from molecular hydrogen transitions can be seen outside the 155-165 nm region, especially at shorter wavelengths between 130-155 nm. However, the molecular emission peaks at 158 and $161 \mathrm{~nm}$ account by far for most of the emission and are therefore considered to be most sensitive to changes in the lamp settings.

Lyman- $\alpha$ and molecular emission fluxes are normalized to $100 \%$ of the total flux of the spectrum in order to visualize trends in the contribution of certain spectral regions. The remaining part of the spectrum is discussed as well. The $2 \sigma$ error is determined from 15 individual measurements performed at identical settings throughout the measurement run. Errors are found to be $\pm 11 \%$ in the total photon flux, $\pm 4 \%$ in the Lyman- $\alpha$ contribution, $\pm 1 \%$ in the molecular emission contribution and $\pm 3 \%$ in the remaining emission contribution.

\subsection{CryoPAD II}

The Cryogenic Photoproduct Analysis Device II (CryoPAD II) is used to experimentally test the MDHL SED calibrations on interstellar ice analogues. The set-up is an upgraded version of the system described by Öberg et al. (2007). Ices are grown on a gold coated "cold finger" that is mounted on top of a UHV chamber $\left(5 \times 10^{-11} \mathrm{mbar}\right)$ through directed deposition. A closedcycle helium cryostat realizes temperatures as low as $17 \mathrm{~K}$ and a temperature regulator (Lakeshore) allows accurate temperature settings. Ice thicknesses are determined using isothermal desorption techniques. Also mounted onto the chamber is the MDHL with a $\mathrm{MgF}_{2}$ window to sample distances of $16 \mathrm{~cm}$. The ice diagnostics upon VUV irradiation are realized both spectroscopically and mass spectrometrically. The light of an Agilent FTIR 660 spectrometer $\left(500-4000 \mathrm{~cm}^{-1}, 2.0 \mathrm{~cm}^{-1}\right.$ resolution) is guided in reflection modus onto a MCT detector to obtain RAIR (reflection absorption infrared) spectra. The entire IR beam path is purged with water-free dry air. Perpendicular to the IR beam, a Pfeiffer QMA200 Quadrupole Mass Spectrometer (QMS) is mounted to sample gas phase species that are emitted from the ice surface. This construction allows the photoprocessing of the ice upon VUV irradiation to be studied in situ and in real time. Details of the methods used have been described in Ioppolo et al. (2014) and Linnartz et al. (2015). 


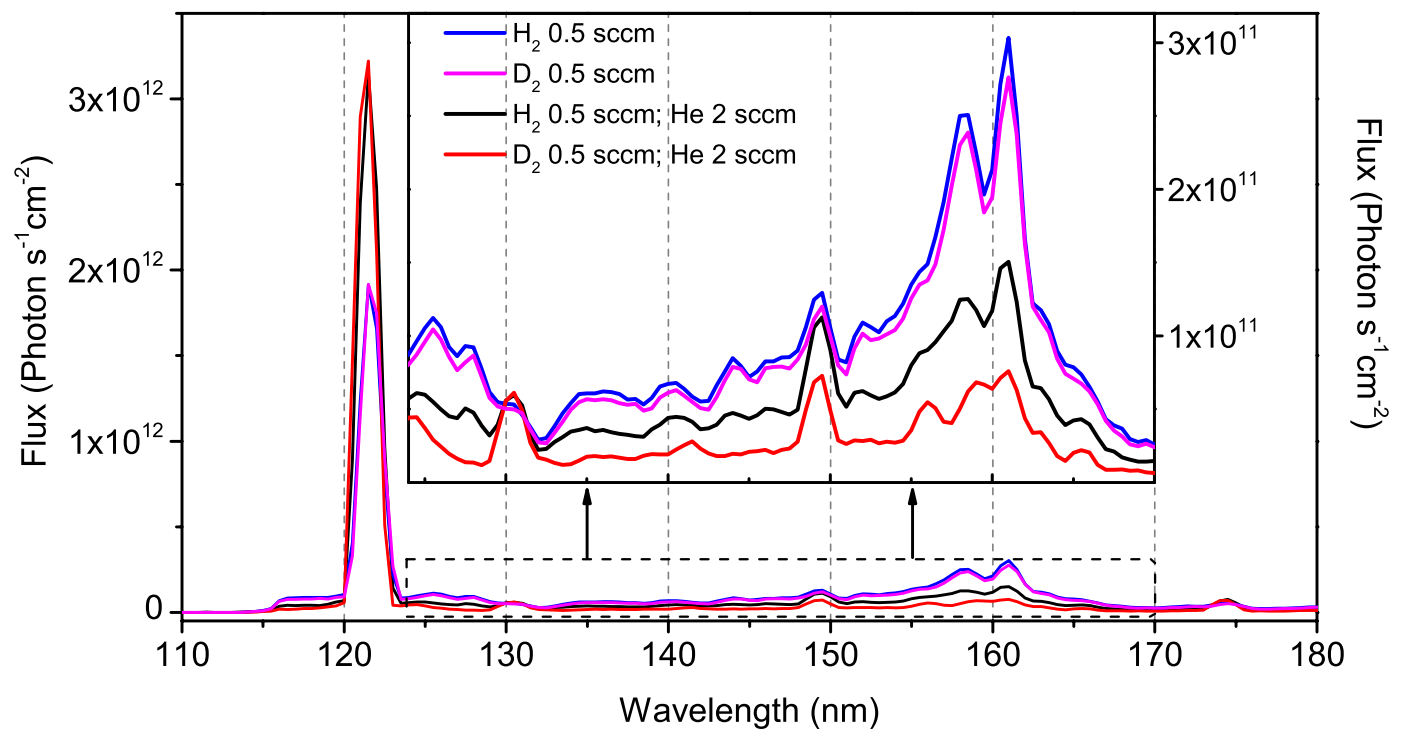

Fig. 3. VUV spectra in the $110-180 \mathrm{~nm}$ range of a T-type geometry MDHL, the $\mathrm{H}_{2} / \mathrm{D}_{2}$ partial pressure is kept at $0.41 \mathrm{mbar}$. The effect of adding helium with the $\mathrm{H}_{2}$ or $\mathrm{D}_{2}$ is clearly shown. Photon flux ( $y$-axis) estimations are based on NIST photodiode measurements. Emission at $\sim 130$, $\sim 149$, and $\sim 175 \mathrm{~nm}$ indicate traces of oxygen and nitrogen.

\section{Results}

\subsection{Photon flux}

The most commonly used settings for either an F- or T-type MDHL are 0.41 mbar pure hydrogen pressure, at an input power of $100 \mathrm{~W}$. We take the output generated by the T-type lamp as standard. Photon fluxes obtained from other experiments are normalized to this value for the majority of the data.

The spectrum of the lamp for these settings is shown in Fig. 3. Following the method described in Sect. 2.3 using a calibrated photodiode, a total VUV photocurrent of $1.6 \pm 0.2 \mu \mathrm{A}$ is measured for the standard T-type lamp at a distance of $31 \mathrm{~cm}$ $\left(\mathrm{MgF}_{2}\right.$ window to photodiode). From this current, a photon flux of $(9 \pm 2) \times 10^{12}$ photons s $\mathrm{s}^{-1}$ is derived. Uncertainties in photo current and total flux determine the error in this value. The Lyman- $\alpha$ contribution to the total output is $36 \%$, the molecular emission between 155 and $165 \mathrm{~nm}$ contributes 19\% to the total output and the remaining emission of the lamp has a contribution of $45 \%$. The remaining emission consists of all the output between 116 and $220 \mathrm{~nm}$, excluding what is present in Lyman- $\alpha$ and the molecular part.

Based on the irradiance measurements performed in Greifswald, assuming a homogenous circular emitting source at the position of the aperture, we have calculated the photon flux. This leads to a total VUV photon flux at the photodiode position of $\sim 1.3 \times 10^{13}$ photons $\mathrm{s}^{-1} \mathrm{~cm}^{-2}$, within a factor of two agreement with the value acquired in the direct flux measurement in Leiden.

\section{2. $\mathrm{H}_{2}$ pressure dependence}

In Fig. 4, the response of an F-type MDHL is plotted as a function of hydrogen pressure. The total flux is normalized with respect to the standard settings of the T-type MDHL. The relative contributions of Lyman- $\alpha$ (120-124 nm), molecular emission $(155-165 \mathrm{~nm})$, and remaining emission is presented as well. Clearly visible at $0.41 \mathrm{mbar}_{2}$ is a maximum in the total flux. For this pressure, the contribution of the Lyman- $\alpha$ band reaches the maximum as well, while the contribution in molecular emission is still significantly high. The remaining emission clearly has a local minimum at this pressure. At higher $\mathrm{H}_{2}$ pressure, above $1 \mathrm{mbar}$, the total flux gradually decreases. It starts increasing again above 4 mbar. Lyman- $\alpha$ gradually decreases to zero for pressures above 0.41 mbar. The molecular emission shows a similar trend up to 4 mbar. At this point the molecular contribution steeply increases again and is responsible for the overall increase in total flux.

In summary: higher $\mathrm{H}_{2}$ pressures (with respect to the standard settings) favour emission in the $160 \mathrm{~nm}$ region, whereas pressures between 0.4-0.8 mbar are better for Lyman- $\alpha$ emission.

\subsection{Mixtures and different gasses}

Adding helium to hydrogen in a MDHL can have a significant influence on the SED and flux (Holländer \& Wertheimer 1994; Benilan et al. 2011; Chen et al. 2014; Cook et al. 2014; Es-sebbar et al. 2015). This is illustrated in Fig. 5A, which shows a decline in the total flux moving to higher concentration of helium admixtures. However, a dependence on the $\mathrm{H}_{2}$ pressure used in the mixture is also seen. The general trend is comparable with the flux variation in pure $\mathrm{H}_{2}$ where flux decreases towards higher pressure. In Fig. 5B, the spectral contribution is depicted for different $\mathrm{H}_{2}$ pressures $(0.41,0.59$, and $0.76 \mathrm{mbar})$. Within the error bars, the spectral contributions measured at different $\mathrm{H}_{2}$ pressures overlap, confirming that in this pressure regime the amount of $\mathrm{He}$ is the dominant source of spectral change.

The addition of He to $\mathrm{H}_{2}$ (and $\mathrm{D}_{2}$ as will be discussed below) significantly alters the spectral emission pattern. By adding $2 \mathrm{sccm}$ of He, the spectral purity of Lyman- $\alpha$ increases by a factor of $\sim 1.65$ from $35-40 \%$ to $60-65 \%$ while the total flux is nearly constant. The helium has a quenching effect on the molecular hydrogen, while increasing the effective Lyman- $\alpha$ flux.

As more $\mathrm{He}$ is added to $\mathrm{H}_{2}$, the Lyman- $\alpha$ and molecular emission contributions reach a maximum and minimum, respectively, and remain at a stable level. In contrast to the spectral contributions, the total photon flux peaks around an $\mathrm{H}_{2}$ : $\mathrm{He}$ 1:2 mixing ratio and slowly decreases for higher amounts of helium.

Further alteration of the MDHL output can be accomplished by changing from $\mathrm{H}_{2}$ to $\mathrm{D}_{2}$ mixed with helium. This results 
N. F. W. Ligterink et al.: Controlling the emission profile of an $\mathrm{H}_{2}$ discharge lamp to simulate interstellar radiation fields

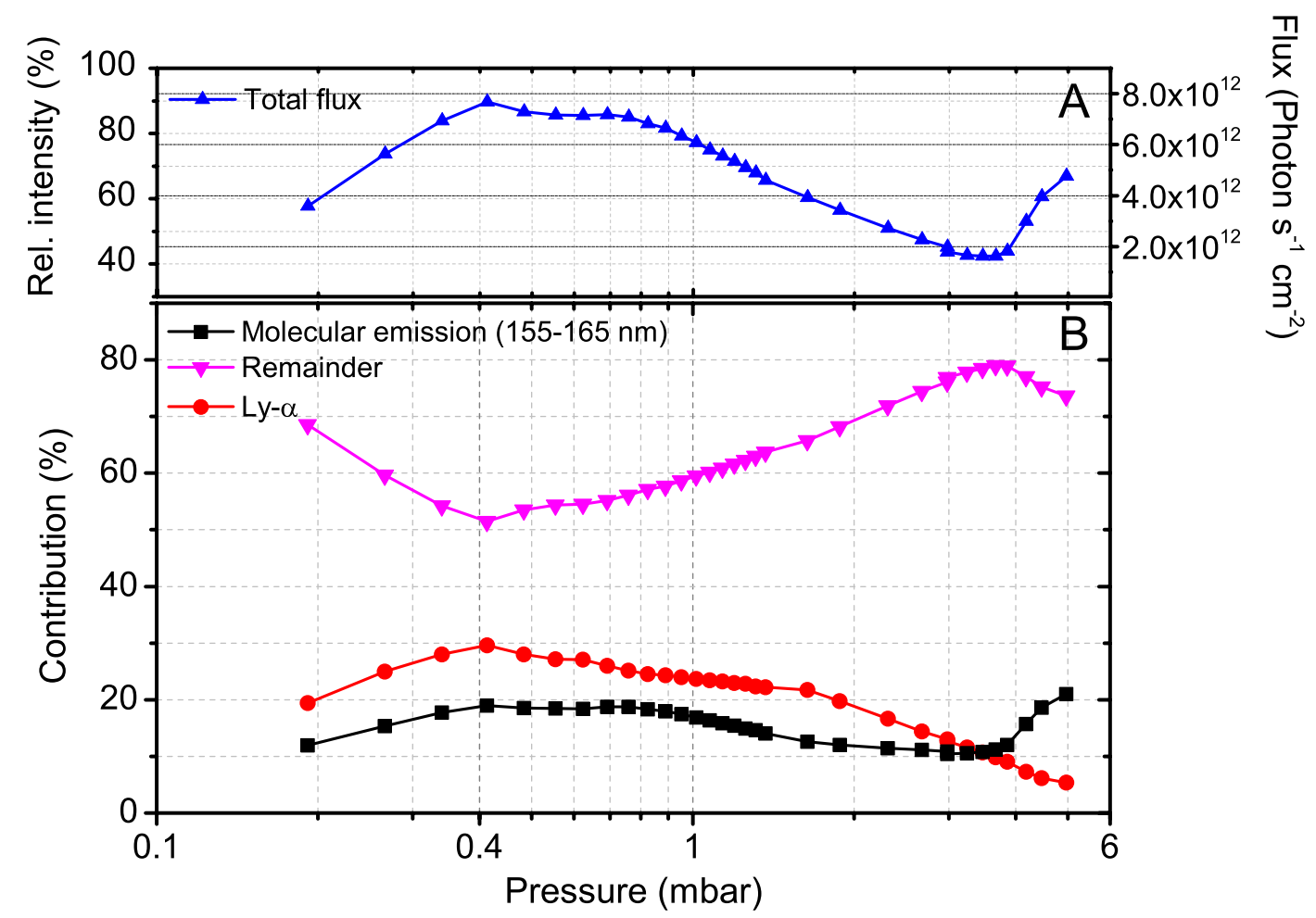

Fig. 4. Total flux A) and spectral contribution B) as a function of $\mathrm{H}_{2}$ pressure dependence of a F-type MDHL for standard settings. The relative intensity (left axis) is normalized to $100 \%$ of the standard T-type MDHL, photon flux (right axis) estimations are based on NIST photodiode measurements.

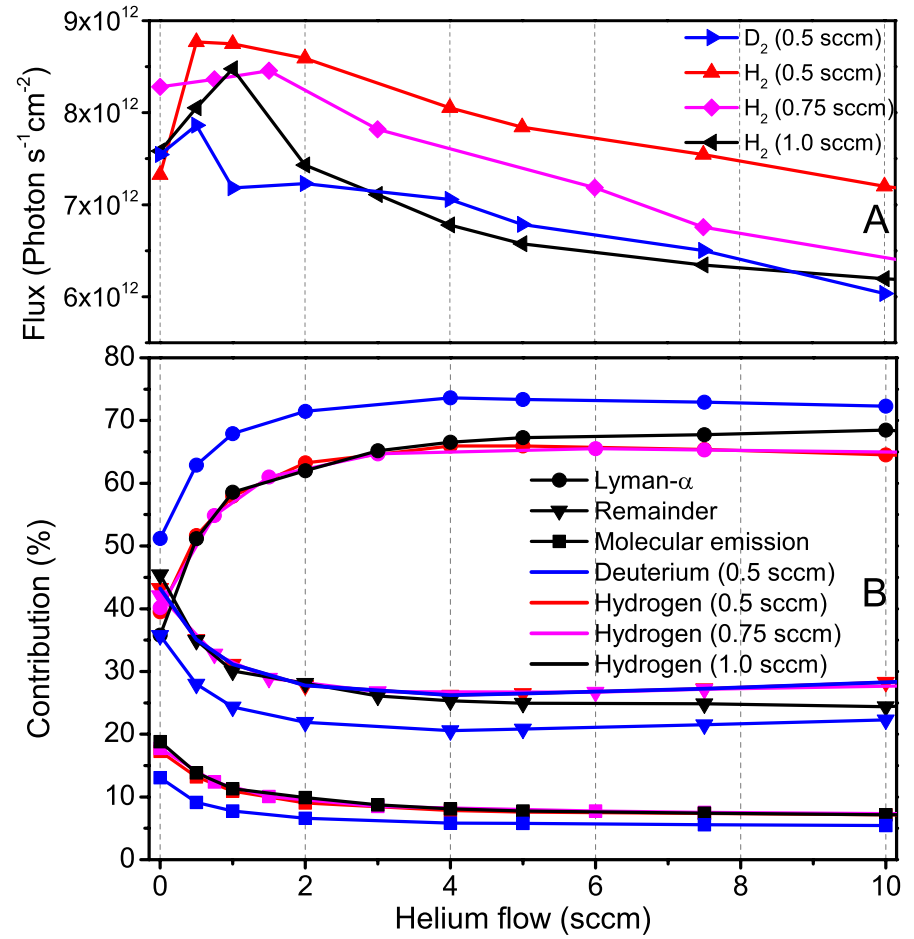

Fig. 5. Flux dependance A) and spectral contribution B) of $\mathrm{H}_{2}$ or $\mathrm{D}_{2}$ as function of helium flow. Different fixed molecular hydrogen flows are shown $(0.5,0.75$, and $1.0 \mathrm{sccm})$, resulting in $0.41,0.59$, and $0.76 \mathrm{mbar}$ as a function of the helium flow rate. Additionally, $\mathrm{D}_{2}$ in $\mathrm{He}$ is depicted (0.5 sccm/0.41 mbar).

in the highest contribution (up to $\sim 75 \%$ ) of Lyman- $\alpha$ and lowest molecular emission and remaining contributions. The photon flux is reduced by roughly $15 \%$ compared to 0.41 mbar hydrogen mixed in helium, however. The corresponding VUV spectra are shown in Fig. 3.

Similar to the $1: 15 \mathrm{H}_{2}$ :He mixing ratio shown in Chen et al. (2014), we find a substantial effect of helium addition to hydrogen. A different mixing range has been evaluated in Es-sebbar et al. (2015), but extrapolating our settings to theirs seems to yield a comparable trend in photon flux decrease. The changing $\mathrm{H}_{2}$ concentration might be the explanation for the increase in Lyman- $\alpha$ contribution. From the extended measurements performed in this article more trends become apparent. At higher ad-mixing of helium, the total flux decreases while the spectral purity of Lyman- $\alpha$ does not increase further. We conclude that the highest observed contribution of Lyman- $\alpha$ emission, is realized for $\mathrm{D}_{2}$ in $\mathrm{He}$.

\subsection{MDHL power dependence}

The microwave (MW) cavity input power determines the strength of the microwave field used in the MDHL, and thus directly influences how intensely the plasma is excited. This may also effect the SED. So far, three studies have been reported that focus on the MW dependency. Cottin et al. (2003) presented flux measurements over a limited range, from 30 to 80 W. Cruz-Diaz (2014) presented data about the peak intensities at Lyman- $\alpha, 157.8 \mathrm{~nm}$ and $160.8 \mathrm{~nm}$, for MW powers ranging from 55 to 90 W. Finally, Es-sebbar et al. (2015) conducted a wavelength dependent study in combination with actinometry of the MW power on $\mathrm{H}_{2}$ :He mixtures. All three of the studies hint towards a linear behaviour between flux and MW input power. This is confirmed in our study. Figure 6 shows the relative output of an F-type MDHL at 0.41 mbar hydrogen versus microwave cavity input power, in the range of 50 to $130 \mathrm{~W}$. Up 


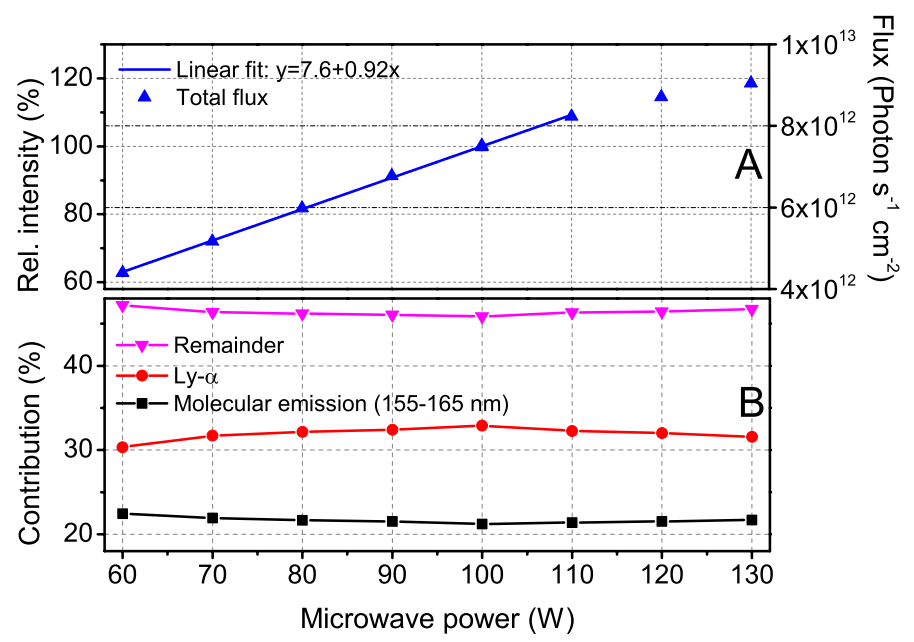

Fig. 6. Relative flux dependance on microwave cavity input power. The total flux is normalized to the $100 \mathrm{~W}$ value. Lyman- $\alpha$, molecular emission and remainder are given as percentages of the total flux. Corrected for time dependent behaviour.

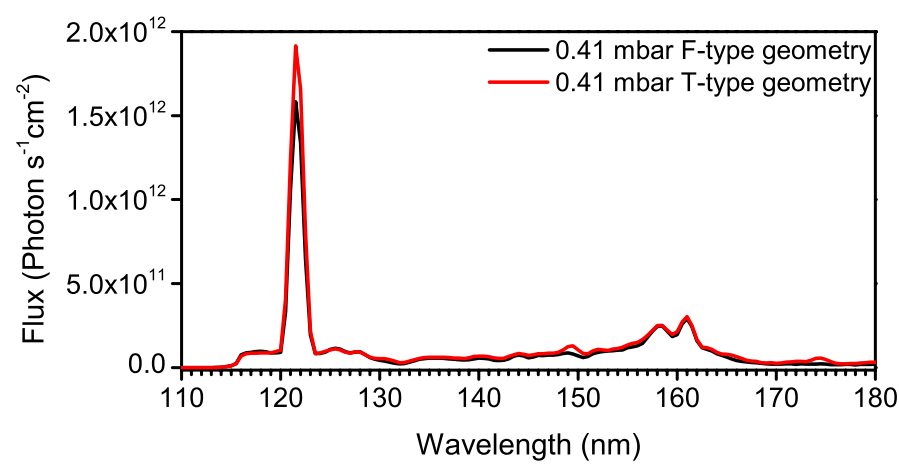

Fig. 7. VUV spectra in the $110-180 \mathrm{~nm}$ range of different geometry MDHLs (F- and T-types) at a molecular hydrogen pressure of 0.41 mbar.

to $110 \mathrm{~W}$, the total photon flux increases linearly with input microwave power. Above $110 \mathrm{~W}$ the increase in flux is not as steep, but still significant. At $130 \mathrm{~W}$, the trend suggests that the flux is reaching a plateau. At this microwave power the air cooling is no longer sufficient and the temperature of the cavity starts increasing. Therefore no higher microwave power measurements were performed. The relative contributions of Lyman- $\alpha$, molecular, and remaining emission are also presented in Fig. 6. As can be seen, the lamp spectrum does not follow the photon flux trend linearly, but the deviations are not substantial. This finding is different from the conclusion derived in Es-sebbar et al. (2015) for $\mathrm{H}_{2}$ :He mixtures. The molecular emission and the Lyman- $\alpha$ contribution have a respective minimum and maximum at $100 \mathrm{~W}$ input power. The relative difference is as big as $10 \%$. In conclusion, tuning the input power between 60 and $120 \mathrm{~W}$ offers a way to tune the photon flux output by up to a factor of 2 . With the change in power small changes in SED are also found.

\subsection{Pumping efficiency in F- and T-type MDHLs}

Comparative measurements between an F- and T-type MDHL have been performed. Under identical settings the output was measured for a number of hydrogen pressures ranging from 0.24 to 0.7 mbar. In Fig. 7 an example is presented for a hydrogen pressure of 0.41 mbar.

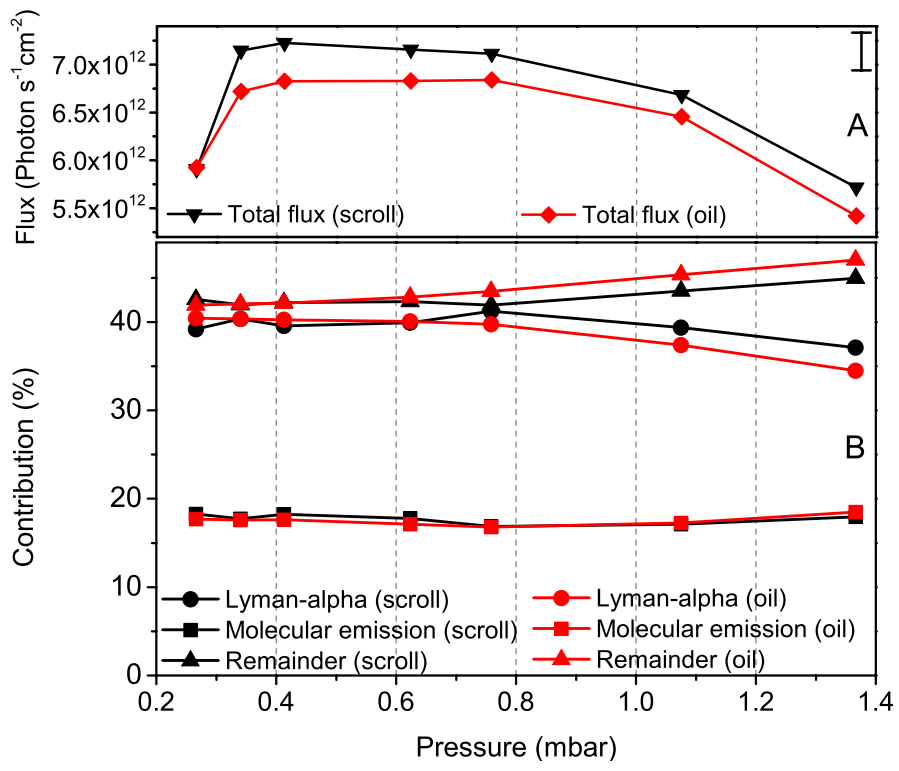

Fig. 8. Lyman- $\alpha$ (circles), molecular emission (squares), remainder (triangles) contribution, and normalized total photon flux (top) for various pressures with different pumps. The oil pump (black) gives a lower photon flux than the scroll pump (red), but the contributions are virtually identical.

The T-type lamp is found to increase the Lyman- $\alpha$ emission by $30 \%$ on average, compared to the F-type MDHL. At the same time the molecular hydrogen emission remains identical for the F- and T-type MDHL. A total photon flux increase is also observed for the T-type lamp, which can be explained by the increase in Lyman- $\alpha$ emission. The increase of this particular atomic hydrogen line was also reported by Chen et al. (2014) for a pressure of 0.4 torr $(0.53 \mathrm{mbar})$. They found an increase in Lyman- $\alpha$ when using a T-type MDHL of more than $100 \%$ compared to an F-type lamp as well as a decrease in molecular emission. These findings are not fully in line with the results in the present study. Chen et al. (2014) suggested that different pumping efficiencies were responsible for the difference in spectral output of the F- and T-type MDHLs. The T-shape geometry ensures that gasses can be more easily evacuated from the MDHL. This in turn limits collisional de-excitation of excited atomic hydrogen and therefore an increase in Lyman- $\alpha$ output is seen. Building on this argument, using different pumping capacities on the same lamp should make it possible to reproduce this effect. This can be achieved by using different types of pumps, for example a scroll pump and rotary vane pump. Respectively, these pumps are able to reach a base pressure in the low $10^{-3}$ and low $10^{-2} \mathrm{mbar}$ region and have a pumping capacity of $285 \mathrm{l} / \mathrm{min}$ and $300 \mathrm{l} / \mathrm{min}$ for air. In addition, it is known that scroll pumps do not pump hydrogen as efficiently as oil-based rotary vane pumps.

Two sets of measurements were performed with an F-type lamp running at various $\mathrm{H}_{2}$ pressures, while being pumped by either a scroll pump or a rotary vane pump. Although the input pressure is identical in both sets of experiments, a pressure difference between the two pumps was observed on the gauge at the pump side. Pressures are between 6 and $60 \%$ lower for the rotary vane pump, confirming that the rotary vane pump removes hydrogen more efficiently from the MDHL than the scroll pump.

The VUV spectra, shown in Fig. 8, show no significant change in the spectral distribution of the lamp output for the two different pumps. Only the total photon flux output is higher for the measurements with the scroll pump. Therefore the pumping 
N. F. W. Ligterink et al.: Controlling the emission profile of an $\mathrm{H}_{2}$ discharge lamp to simulate interstellar radiation fields

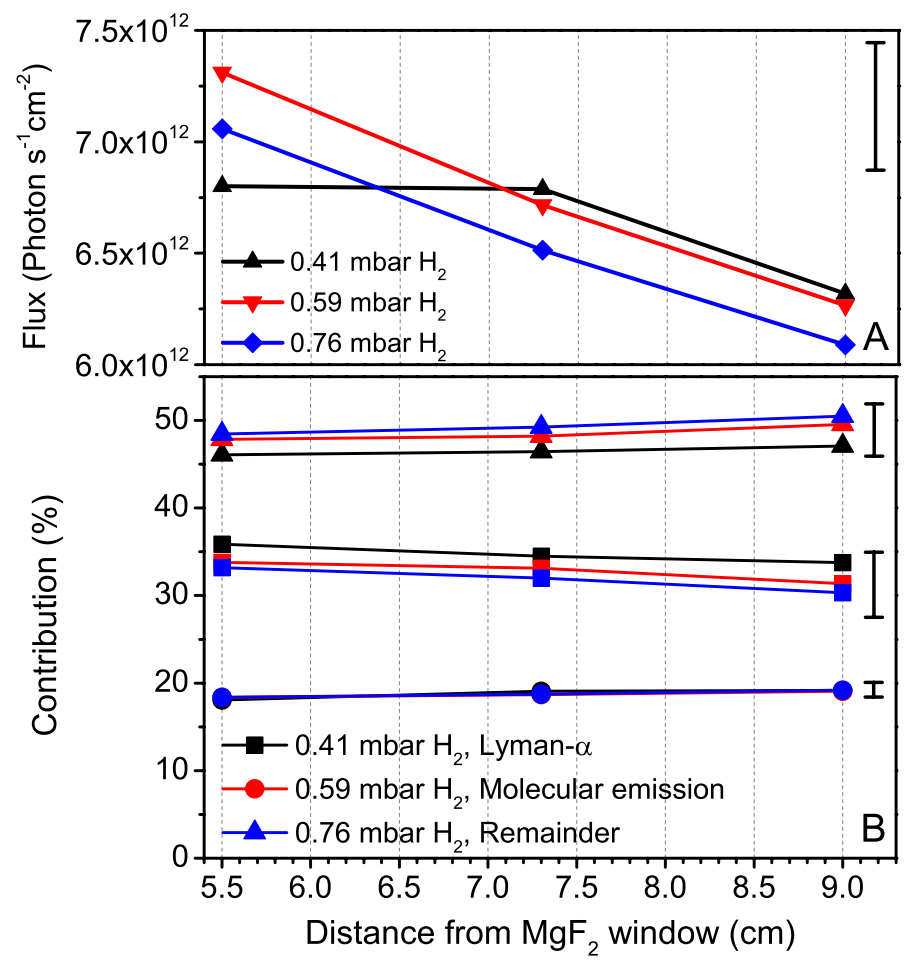

Fig. 9. Variations in spectral output depending on cavity location, 0.41 mbar (black), 0.59 mbar (red), and 0.76 mbar (magenta) $\mathrm{H}_{2}$ flow pressure. Lyman- $\alpha$ (squares), molecular emission (circles), and remainder of the VUV spectrum are shown (triangles).

efficiency cannot explain the differences in spectral distribution between an F- and T-type lamp. It is not fully clear what causes this difference in Chen et al. (2014), because for similar settings the effect is not found here. It could be caused by the slightly different geometries of the lamps.

In conclusion, choosing a T-type cavity can be beneficial when higher Lyman- $\alpha$ and total flux is required. Although the spectral distribution is not expected to change for different pumping efficiencies, the total photon flux can be slightly affected. Therefore, when running a series of experiments, the same pumping system should always be used.

\subsection{Distance dependence}

In this study measurements are performed to determine the influence of the microwave cavity positioning on the output spectrum. The measurements are done at cavity positions of 9.0, 7.3, and $5.5 \mathrm{~cm}$ from the $\mathrm{MgF}_{2}$ window for $\mathrm{H}_{2}$ flow pressures of $0.41,0.59$, and 0.76 mbar (see Fig. 9). The overall trend is that the total photon flux decreases slightly while moving the cavity away from the $\mathrm{MgF}_{2}$ window. Additionally, the spectral energy distribution does show minor changes as well. The percentagewise contribution of Lyman- $\alpha$ increases and the molecular emission contribution decreases when the cavity is moved towards the $\mathrm{MgF}_{2}$ window. Although this is small and within the error, this effect is observed for all pressures studied.

Although it is a small effect (for the distances studied here), the change in photon flux is interesting. The limited data set available scales with an isotropic $r^{-2}$ decrease, suggesting that the MW cavity defines a point source. The change in spectral output may be related to a higher column density of rest gasses in between the plasma and window when increasing the distance, affecting the SED in a wavelength dependent way. At this stage it is not possible to say more; the overall effects are small.

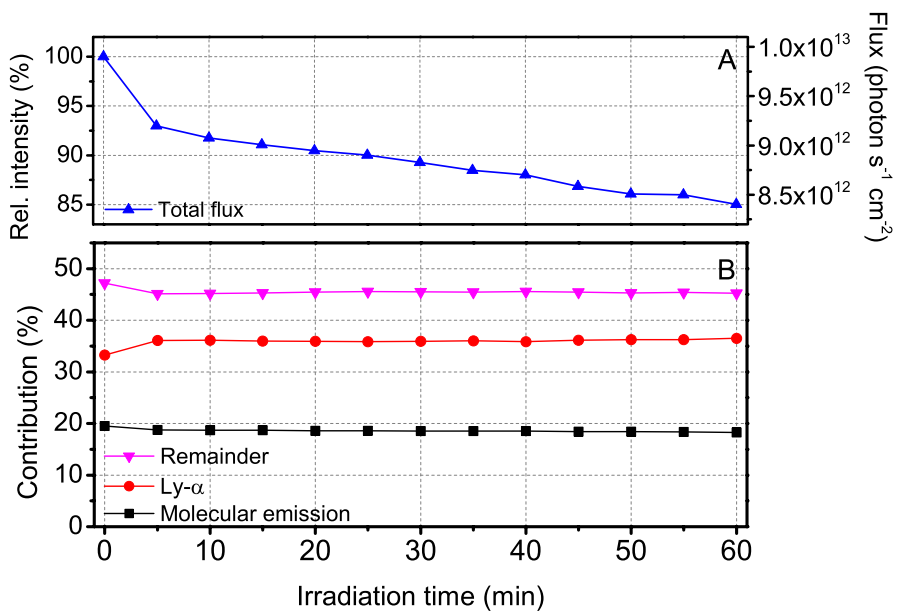

Fig. 10. Relative flux decrease over time, combined with the relative contribution of Lyman- $\alpha$, molecular, and remaining emission for standard MDHL settings, using a T-type lamp.

Nevertheless, as the position does matter, keeping the cavity on a fixed position during a set of experiments is recommended.

\subsection{Practical issues}

In addition to the systematic dependences discussed before, there are also a number of very practical issues that should be taken into account when operating the MDHL. The most prominent practical issues running MDHLs involve stability over time (Sect. 3.7.1) and the effect of degradation of the $\mathrm{MgF}_{2}$ window (Sect. 3.7.2).

\subsubsection{MDHL stability over time}

In many of the previous studies using MDHLs it was found that these exhibit changes in the output photon flux over operating time. Two important contributors to this effect are thermal settling of the lamp and degradation and pollution of the transmission window (Warneck 1962; Chen et al. 2014). To determine the stability of our lamp spectrum over time, a T-type MDHL was run at $0.41 \mathrm{mbar}_{2}$ for an hour while taking a spectrum every 5 min (see Fig. 10). The first spectrum was taken immediately after turning on the MDHL. In the first 5 min a drop in total photon flux is seen, after which the output linearly decreases. This decrease is mainly caused by a quick drop in molecular emission. The linear decrease in flux is similar to that reported by Chen et al. (2014); however, no stable region is reached, possibly owing to the shorter duration of the experiment (one hour in this work and two and a half hours in Chen et al. 2014).

More interesting are the Lyman- $\alpha$ and molecular emission contributions to the total flux. These are found to remain at a stable level after the first $5 \mathrm{~min}$ of lamp operation, which guarantees that spectral properties of the lamp are directly proportional to its photon flux.

This result is surprising, since Warneck (1962) found that the contribution of the Lyman- $\alpha$ component decreases more rapidly than the molecular contribution. This is possibly due to differences in the way the SEDs are determined. Warneck (1962) made use of a nickel photocell to detect the 120-130 nm range and ozone actinometry for the $135-185 \mathrm{~nm}$ region. These broadband techniques are not suitable for distinguishing wavelength dependences and consequently only provide information on the overall flux. For general lamp operation starting the irradiation 
experiments after the lamp has settled for 10 to $15 \mathrm{~min}$ is recommended. In this way the photon flux and spectral fluctuations do not play a dominant role in the experiment. After this period the spectral contributions will remain stable and therefore it is sufficient to only trace variations in the photonflux.

\subsubsection{Effects on the $\mathrm{MgF}_{2}$ window}

As already suggested in paragraph 3.7.1, pollution of the $\mathrm{MgF}_{2}$ window can have a big influence on the output of the MDHL. Carbon products formed in the plasma can deposit on the MDHL side of the window. Especially if the VUV lamp is being pumped with an oil-based pump, there will be oil vapours in the system that can be processed by the plasma and deposited as a residue on the window. On the vacuum chamber side, gas molecules can be adsorbed on the window, and residual gas molecules will be present in the vacuum chamber. When the lamp is in operation these molecules can be processed by the VUV field to form a residual layer. Es-sebbar et al. (2015) showed a decrease of $50 \%$ in transmission of the $\mathrm{MgF}_{2}$ window after 8 hours of irradiation, which was ascribed to the deposition of trace species. We do not observe such a drastic decrease in transmission. However, this discrepancy could be explained by a difference in measurement technique. A third effect is the processing of the window itself. The harsh VUV output of the lamp causes structural changes of the material over time. This was shown by Warneck (1962) for LiF, and it is also likely to happen for $\mathrm{MgF}_{2}$, although on a different time scale. These two effects, pollution and window processing, can be responsible for diminishing the flux and may cause spectral changes over longer periods of time.

It should be noted that in the Greifswald measurements, the window degradation has not been explicitly taken into account, as this would imply that the window had to be removed regularly. Instead, we expect that degradation effects along a series of measurements, focussing on one specific parameter, are negligible or much less relevant than the effect of the changing parameter under investigation.

\subsection{CO photodepletion measurements}

Laboratory experiments have shown that photodesorption offers an effective non-thermal mechanism that explains the detection of molecular gas phase species in low temperature regions in the ISM where these species are expected to be fully frozen out. However, the exact numbers vary between different groups, even when experimental procedures are largely identical. This has become clear, particularly for the photodesorption yield obtained for pure CO ice. Öberg et al. (2007), Muñoz Caro et al. (2010), and Chen et al. (2014) provide values of $(2.7 \pm 1.3) \times 10^{-3}$, $(35 \pm 5) \times 10^{-3}$, and $(212 \pm 3) \times 10^{-3}$ molecules per incident photon, respectively. These measurements have been performed with broadband MDHLs. In a monochromatic and wavelength dependent study Fayolle et al. (2011) showed that CO photodesorption occurs predominantly at longer wavelengths between 135 and $165 \mathrm{~nm}$, following a desorption induced by electronic transition (DIET) mechanism. For wavelengths around Lyman- $\alpha$ the photodesorption is much less efficient. Therefore, different SED characteristics, specifically the Lyman- $\alpha$ to molecular emission band ratio, will definitely influence the overall photodesorption rate that follows from the MDHL experiments. This is illustrated in Fig. 11 where the corresponding SEDs of two well-characterized lamp settings are used to derive photodesorption rates.

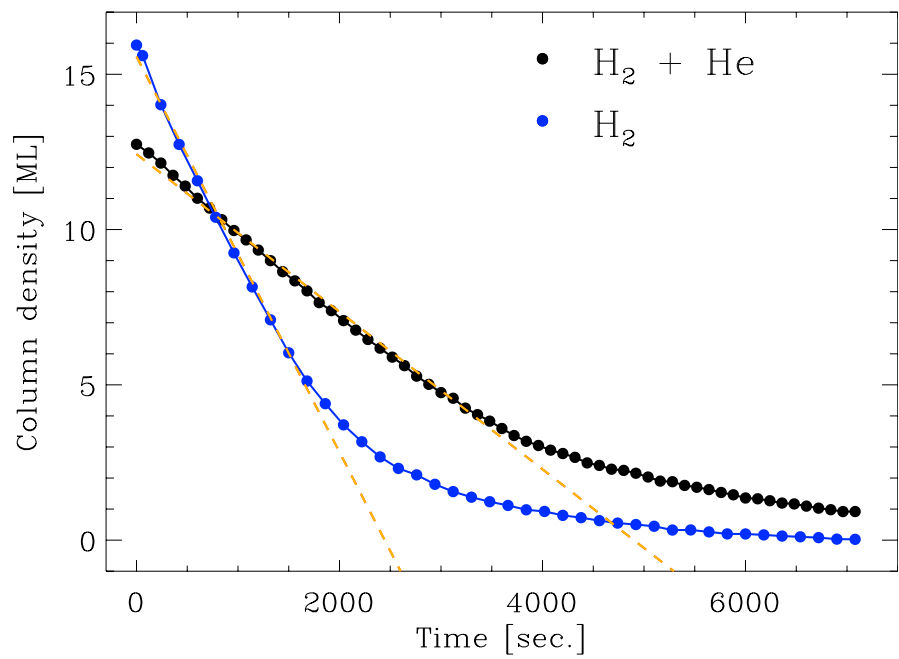

Fig. 11. $\mathrm{CO}$ photodesorption of ices irradiated with either pure $\mathrm{H}_{2}$ at 0.41 mbar (blue) or $\mathrm{H}_{2}: \mathrm{He} 1: 3$ (black) at 2.13 mbar.

Using CryoPAD II, a T-type MDHL is operated at $100 \mathrm{~W}$ and either 0.41 mbar of pure $\mathrm{H}_{2}$ or a $1: 3 \mathrm{H}_{2}: \mathrm{He}$ gas mixture, at a pressure of $2.13 \mathrm{mbar}$, is used. The SEDs for these settings comprise $36 \%$ and $67 \%$ of Lyman- $\alpha$ and $19 \%$ and $8 \%$ molecular emission, respectively, while the total flux for both settings is similar (less than $\sim 0.5 \times 10^{12}$ photons s ${ }^{-1} \mathrm{~cm}^{-2}$ difference, or about $6 \%$, in the calibrated measurements). The vacuum UV photons impinge on thin ice layers (13 or 16 MonoLayers (MLs), $1 \mathrm{ML}=10^{15}$ molecules) of pure CO deposited on top of a $17 \mathrm{~K}$ gold surface; the layers are thick enough to exclude surface effects. A typical experiment lasts $2 \mathrm{~h}$. The ongoing photodepletion (the combined effect of photodesorption and photochemistry) of the $\mathrm{CO}$ ice over time is subsequently tracked by the decrease in the $2142 \mathrm{~cm}^{-1} \mathrm{CO}$ band.

Taking thicknesses of $5 \mathrm{ML}$ or more as the regime where surface effects can be neglected, the linear fits allow a photodepletion rate to be derived. As expected from the work by Fayolle et al. (2011), an ice irradiated with light from the MDHL running on the $\mathrm{H}_{2}$ :He mixture (suppressed $160 \mathrm{~nm}$ emission) photodepletes less rapidly than the ice irradiated with pure $\mathrm{H}_{2}$ that exhibits a molecular emission that is roughly two times bigger. Obviously, this photodepletion value is different for the different lamp settings, fully in agreement with the main findings of the work presented in this paper. It also may explain, at least to some extent, the deviations found in the different photodesorption studies in agreement with Chen et al. (2014). It is for this reason that we stress how important it is to record the SEDs in situ. Other artefacts might also be at play; it is likely that other photo induced effects affect the final values, (wavelength dependent) chemical processes forming $\mathrm{CO}_{2}$, for example, that in turn may photodesorb (wavelength dependent, see Fillion et al. 2014). The two curves in the figure yield a photodepletion rate of $(6 \pm 3) \times 10^{-3}$ and $(3 \pm 1) \times 10^{-3} \mathrm{ML} \mathrm{s}^{-1}$ for $\mathrm{H}_{2}$ and the $\mathrm{H}_{2}$ :He mixture, respectively. It will take a greater effort to bring all experimental numbers in line, but obviously an important uncertainty has now been characterized.

Besides quantitative issues, a changing SED may also affect the outcome of many chemical studies performed over the past decades; different molecules may react resonantly with different emission bands, and particularly higher energetic photons may cause bonds to cleave more easily. With the information provided here such dependencies can now be studied relatively easily. 
N. F. W. Ligterink et al.: Controlling the emission profile of an $\mathrm{H}_{2}$ discharge lamp to simulate interstellar radiation fields

Table 1. Parameter influences.

\begin{tabular}{cccc}
\hline \hline Influence & $\begin{array}{c}\text { Effect on SED } \\
(\text { Lyman- } \alpha)\end{array}$ & $\begin{array}{c}\text { Effect on SED } \\
\text { (molecular emission) }\end{array}$ & $\begin{array}{c}\text { Effect on flux } \\
\left(\text { photon } \mathrm{cm}^{-2} \mathrm{~s}^{-1} \text { ) }\right.\end{array}$ \\
\hline Pressure & Large effect $\sim 30 \%$ & Large effect $\sim 15 \%$ & Large effect $\sim 30 \%$ \\
Power & Limited effect $\sim 3 \%$ & Limited effect $\sim 3 \%$ & Large effect $\sim 50 \%$ \\
Helium & Large effect $\sim 100 \%$ & Large effect $\sim 50 \%$ & Limited effect $\sim 5 \%$ \\
Deuterium & Moderate effect $\sim 10 \%$ & Moderate effect $\sim 10 \%$ & Moderate effect $\sim 15 \%$ \\
F- vs. T-type MDHL & Moderate effect $\sim 20 \%$ & Limited effect & Moderate effect $\sim 20 \%$ \\
Cavity & Limited effect $\sim 3 \%$ & Limited effect $\sim 3 \%$ & Limited effect \\
Time & No effect & No effect & Limited/Moderate effect $\sim 7 \%$ \\
\hline
\end{tabular}

\section{Conclusions}

In this work the influence of various running conditions on both flux and SED of the microwave discharge hydrogen-flow lamp has been studied. The main findings are summarized in Table 1. The conclusions of this work are:

1. The effects of hydrogen pressure, ad-mixing helium, exchanging hydrogen for deuterium, microwave power, pumping efficiency, cavity position, and practical issues such as stability over time need to be taken into account.

2. The relative contribution of Lyman- $\alpha$ to the emission profile of the MDHL can be increased by up to $65 \%$ when helium is added to the hydrogen. Further spectral purity of Lyman- $\alpha$ of up to nearly $75 \%$ can be achieved by exchanging hydrogen for deuterium. Alternatively, high percentages of molecular emission can be generated by increasing the hydrogen pressure.

3. Recommendations are given on how to operate the MDHL at the highest efficiency and consistency. They include allowing sufficient time for the settling of the lamp, using a fixed cavity position, and accounting for pollution on the lamp window.

4. Variations in the SED can, to a certain extent, explain the differences in the literature among different laboratory experiments. However, it seems unlikely that it will explain order of magnitude differences.

5. It is important to characterize the SED and photon flux in situ, preferably with a combination of monochromator and calibrated photodiode.

Acknowledgements. We thank the INP Greifswald for making their UV/VUV set-up available to this research and thank Peter Holtz for his invaluable help in the laboratory. We thank Jean-Hugues Fillion and Mathieu Bertin (UPMC Paris) for many helpful discussions. We also thank Alan Heays and Gleb Fedoseev (University Leiden). Finally we would like to thank the referee of this article, Joseph Nuth, whose open report and suggestions were very helpful. The research leading to these results has received funding from the European Union Seventh Framework Programme (FP7/2007-2013) under grant agreement No. 316216.

\section{References}

Baratta, G. A., Leto, G., \& Palumbo, M. E. 2002, A\&A, 384, 343 Benilan, Y., Gazeau, M.-C., Es-Sebbar, E.-T., et al. 2011, in EPSC-DPS Joint Meeting, 1317

Chen, Y.-J., Chuang, K.-J., Muñoz Caro, G. M., et al. 2014, ApJ, 781, 15

Cook, A. M., Mattioda, A. L., Quinn, R. C., et al. 2014, ApJS, 210, 15

Cottin, H., Moore, M. H., \& Bénilan, Y. 2003, ApJ, 590, 874

Cruz-Diaz, G. A. 2014, Ph.D. Thesis, Centro de Astrobiología, Universidad Autonoma de Madrid

Cruz-Diaz, G. A., Muñoz Caro, G. M., Chen, Y.-J., \& Yih, T.-S. 2014a, A\&A, 562, A119

Cruz-Diaz, G. A., Muñoz Caro, G. M., Chen, Y.-J., \& Yih, T.-S. 2014b, A\&A, 562, A 120

Davis, D., \& Braun, W. 1968, Appl. Opt., 7, 2071

Es-sebbar, E.-T., Bénilan, Y., Fray, N., et al. 2015, ApJS, 218, 19

Fayolle, E. C., Bertin, M., Romanzin, C., et al. 2011, ApJ, 739, L36

Fayolle, E. C., Bertin, M., Romanzin, C., et al. 2013, A\&A, 556, A122

Fillion, J.-H., Fayolle, E. C., Michaut, X., et al. 2014, Faraday Discussions, 168, 533

Foest, R., Kindel, E., Lange, H., et al. 2007, Contrib. Plasm. Phys., 47, 119

Fulvio, D., Brieva, A. C., Cuylle, S. H., et al. 2014, Appl. Phys. Lett., 105, 014105

Gerakines, P. A., Schutte, W. A., \& Ehrenfreund, P. 1996, A\&A, 312, 289

Groth, W. 1937, Z. Phys. Chem., 37, 307

Henderson, B. L., \&, Gudipati, M. S. 2015, ApJ, 800, 66

Holländer, A., \& Wertheimer, M. 1994, J. Vac. Technol. A, 12

Ioppolo, S., Fedoseev, G., Minissale, M., et al. 2014, Phys. Chem. Chem. Phys. (Incorporating Faraday Transactions), 16, 8270

Islam, F., Baratta, G. A., \& Palumbo, M. E. 2014, A\&A, 561, A73

Lange, H., Foest, R., Schafer, J., \& Weltmann, K.-D. 2009, Plasma Sci., IEEE Transactions on, 37, 859

Leto, G., \& Baratta, G. A. 2003, A\&A, 397, 7

Linnartz, H., Ioppolo, S., \& Fedoseev, G. 2015, Inter. Rev. Phys. Chem., 34, 205

Loeffler, M. J., Baratta, G. A., Palumbo, M. E., Strazzulla, G., \& Baragiola, R. A. 2005, A\&A, 435, 587

Muñoz Caro, G. M., Meierhenrich, U. J., Schutte, W. A., et al. 2002, Nature, 416, 403

Muñoz Caro, G. M., Jiménez-Escobar, A., Martín-Gago, J. Á., et al. 2010, A\&A, 522, A108

Öberg, K. I., Fuchs, G. W., Awad, Z., et al. 2007, ApJ, 662, L23

Warneck, P. 1962, Appl. Opt., 1, 721

Watanabe, N., Mouri, O., Nagaoka, A., et al. 2007, ApJ, 668, 1001

Westley, M. S., Baragiola, R. A., Johnson, R. E., \& Baratta, G. A. 1995, Nature, 373,405 